\title{
ENSEÑANZA DEL EMPRENDIMIENTO \\ en la educación superior (diseño de una escala, análisis factorial y confiabilidad)
}

Virginia Guadalupe López Torres Luis Ramón Moreno Moreno Sósima Carrillo

Universidad Autónoma de Baja California

RECIBIDO: 8 DE MAYO DE 2017 ACEPTAdO: 28 DE AGOSTO DE 2017

\section{RESUMEN}

Ta educación en emprendimiento es una 1 estrategia que puede contribuir a que los futuros egresados de la educación superior creen fuentes de empleo y/o, al menos, se autoempleen. El objetivo de este trabajo es realizar el análisis factorial exploratorio de una escala que determine los niveles de educación en emprendimiento que recibe el estudiante universitario. Para ello, primeramente se construyó la escala con base en la revisión del estado del arte, después se realizó un estudio piloto $y$, finalmente, con los datos obtenidos, se determinó su fiabilidad y validez. La muestra estuvo compuesta por sesenta y tres personas inscritas en la educación superior en instituciones ubicadas en la ciudad de Ensenada, Baja California. Los resultados muestran un alfa de Cronbach $>0.9$ en todos los factores. El análisis factorial mostró dos factores que explicaron el $76.919 \%$ de la varianza. Se concluye que las propiedades psicométricas de la escala son adecuadas para medir la educación en empren- 
dimiento de los estudiantes universitarios.

Palabras clave: educación en emprendimiento, estudiantes universitarios, competencias.

\section{Abstract}

ntrepreneurship edu-
cation is a strategy
that can help future graduates of higher education to create sources of employment and/or at least selfemployment. The objective of this work is to perform the exploratory factorial analysis of a scale that determines the levels of entrepreneurial education that the university student receives. For this purpose, the scale was first constructed based on review of the state of art, then a pilot study was carried out, and finally with the data obtained its reliability and validity were determined. The sample consisted of sixty-three people enrolled in higher education in institutions located in the city of Ensenada, Baja California. The results show a Cronbach's alpha $>0.9$ in all factors. The factorial analysis showed two factors that explained $76.919 \%$ of the variance. It is concluded that the psychometric properties of the scale are adequate to measure entrepreneurship education of university students.

Keywords: entrepreneurship education, university students, educational competencies.

\section{INTRODUCCIÓN}

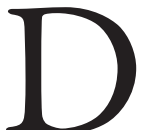
e acuerdo con datos del Instituto Nacional de Estadística y Geografía (Inegi) (2017), en México, en los últimos dos años el salario promedio en la industria manufacturera por hora, se ubicó en 2.3 dólares; la industria alimentaria y la fabricación de prendas de vestir tienen el salario más bajo (1.6 dólares/hora), mientras que la industria dedicada a la fabricación de productos derivados del petróleo y el carbón, así como la industria química son las que otorgan los salarios más altos (6.3 y 4.1 dólares/ hora, respectivamente).

La figura 1 ilustra los salarios por hora de Chile, Estados Unidos y México durante el periodo de 2015 a febrero de 2017, en donde el promedio indica que en Estados Unidos se tiene el mayor salario por hora con 20.2 dólares, seguido de Chile con 4.3 y México con 2.3 dólares, lo que implica que el salario por hora en el país representa $53 \%$ de lo que se paga en Chile y $11 \%$ respecto al salario de Estados Unidos.

Según Campos (2015, p. 95), $13.1 \%$ de la población económicamente activa (PEA) tiene un salario mínimo o menor, lo cual representa a cerca de 6.5 millones de trabajadores. Asimismo, destaca que:

La estructura de salarios no ha cambiado radicalmente desde 2005. Alrededor de $13 \%$ gana un salario mínimo o menos, y alrededor de $26 \%$ gana entre uno y dos salarios mínimos... se resalta que México es el país con el menor salario mínimo en la región una vez que se ajusta la paridad de compra entre países. El país más similar a México en términos de Desarrollo Humano y más cercano en el salario mínimo es Uruguay. El salario mínimo se tendría que aumentar 51\% en México para estar al mismo nivel que en Uruguay (Campos, 2015). 
Flores y Salas (2015), con base en varios autores, señalan que “... la remuneración es la base de la calidad del empleo debido a que es el eje para el desarrollo de los trabajadores" (p. 93). En tal sentido, el empleo en México puede considerarse de baja calidad, dado que no es suficiente para generar bienestar en el corto plazo y estabilidad en el largo plazo.

Otro escenario relevante es el que muestra que de los casi dos millones de desempleados que existen en México, casi la mitad (953 000 personas) tienen un grado de educación media superior o superior, según los datos más recientes del Inegi (2017). De 2013 a 2016, en promedio, $41.50 \%$ de los mexicanos desempleados cursaron el nivel medio superior o una licenciatura (son profesionistas), pero no encuentran un trabajo en la estructura económica del país. Se destaca que el porcentaje más alto, se presentó en el último trimestre, ubicándose en $46.92 \%$ y su tendencia es al alza (véase figura 2).

Este escenario es muy similar al que describen Munuera y Gómez (2016) en España, donde el porcentaje de paro

Figura 1. Salario por hora en la industria manufacturera (dólares).

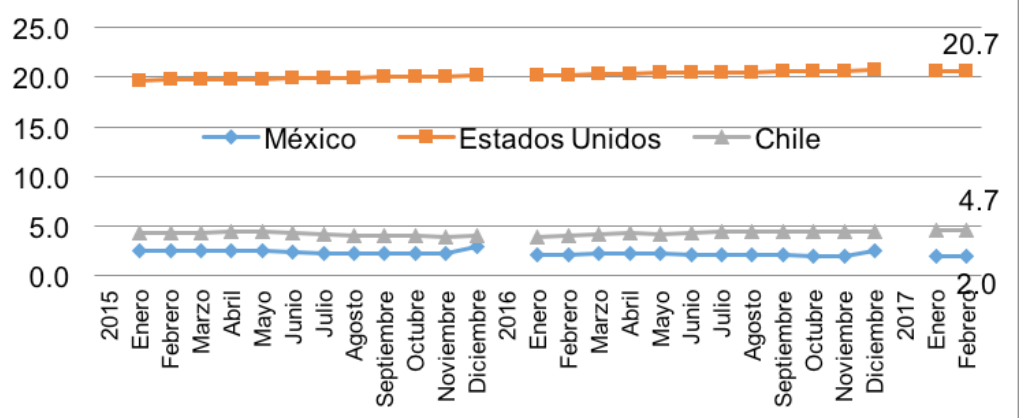

Fuente: elaboración propia con base en datos del Inegi (2017).

Figura 2. Población desocupada.

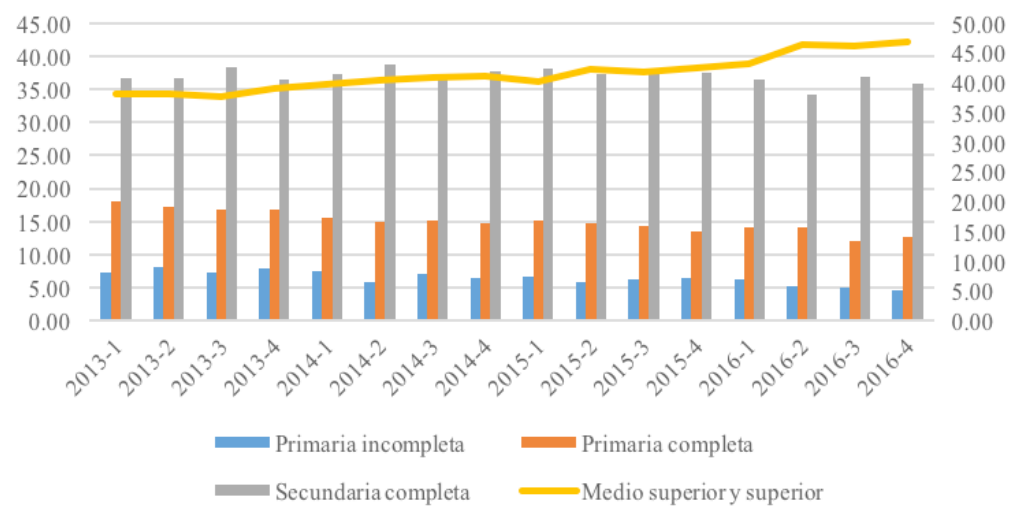

Fuente: elaboración propia con base en datos del Inegi (2017).

en este segmento se ubica en $49.2 \%$, cifra que refleja una difícil realidad que demanda de los universitarios una mejor preparación para afrontar su futuro profesional. En tal sentido, estos autores argumentan que hoy en día las universidades deben ser emprendedoras, lo cual significa que deben desarrollar en su matrícula competencias de innovación y emprendimiento como habilidades mínimas, para que puedan emprender acciones en su contexto que cambien su devenir profesional con suficiente autonomía.

Al considerar este escenario, el presente estudio tiene 
como propósito diseñar una escala que permita medir la enseñanza del emprendimiento (EE), a partir de dos dimensiones: docente (D) y proceso de enseñanza-aprendizaje (PEA). Asimismo, esta escala es valorada utilizando el análisis factorial exploratorio y midiendo su fiabilidad.

En consecuencia, el estudio se diseña con cuatro apartados: en el eje teórico, se describen y analizan los estudios previos realizados; en el método de investigación, se ilustran las definiciones operativas de las dimensiones, los ítems, la población y el estudio piloto; en el apartado de resultados, se presentan los cálculos y medidas resultantes de aplicar el análisis factorial exploratorio y su medición de fiabilidad; $y$, finalmente, se emiten las conclusiones y limitantes de la investigación, la cual cabe destacar que se encuentra en su etapa inicial.

\section{MARCo TEÓRICO}

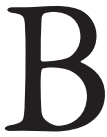
uendía-Martínez y Carrasco (2013) destacan que la actividad emprendedora significa el vehículo para mejorar la calidad de vida de la población, así como un medio para hacer sostenible la economía y el medioambiente.

Para Simón (2013), el emprendimiento es un constructo polisémico que "desde la visión ontológica es inherente a la esencia del ser humano, pues está presente en cada una de las acciones que éste desarrolla para buscar la transformación y mejorar sus condiciones de vida" (p. 161), es decir, todos tenemos el potencial de ser emprendedores. Además, Simón cita a Dehter (2001) para afirmar que nacemos emprendedores y que, a través de la educación, obtenemos herramientas que nos permiten materializar nuestras ideas sin importar el campo de nuestra actividad intelectual y profesional, además de que, al mismo tiempo, mejoramos nuestras aptitudes para emprender.

Complementariamente, Toca (2010) señala que la habilidad para emprender no se encuentra desarrollada por igual en todos los individuos, por lo que debe ser enseñada. Estos argumentos dejan ver que, a través de la educación, puede potenciarse el espíritu emprendedor de cada ser humano. Es importante destacar que el fenómeno del emprendimiento, debe entenderse como la combinación entre la actitud y la capacidad de la persona, binomio que le permite llevar a cabo nuevos proyectos de cualquier índole (SanabriaRangel, Morales-Rubiano y Ortiz-Riaga, 2015).

Restrepo, Tapasco y Vidarte (2016) establecen dos tipos de emprendimiento: el tradicional y el dinámico. El primero es aquel que ingresa en un mercado de producción de bienes que ya se comercializan, con la creencia de que posee características que le permitirán superar la competencia; mientras que el segundo es aquel que posee una visión global, resuelve o crea necesidades en el mercado y se caracteriza por un alto potencial de crecimiento rápido, rentable y sostenible. Está basado en la innovación y tiene la capacidad de generar y capturar un valor extraordinario.

Aunque muchos autores enfatizan en el desarrollo de emprendimientos económicos, Toca (2010) lo hace en la necesidad de ampliar su alcance como campo de conocimiento, considerando que es transversal y transdisciplinario. 
En tal sentido, el reto para las universidades es la formación de individuos capaces de iniciar proyectos de distinta índole (económicos, políticos, públicos o sociales), de propiciar el cambio y el crecimiento de beneficios colectivos o, incluso, de asumir riesgos moderados y calculados, como el que implica la creación de una empresa (Ecotec, 2007; en Sanabria-Rangel, Morales-Rubiano y Ortiz-Riaga, 2015), pero sin limitarse a esa alternativa.

De acuerdo con la Organización de las Naciones Unidas para la Educación, la Ciencia y la Cultura (Unesco) (1998),

la educación tiene que adaptar sus estructuras y métodos de enseñanza a las nuevas necesidades. Pues se trata de pasar de un paradigma centrado en la enseñanza y la transmisión de conocimientos a otro centrado en el aprendizaje y el desarrollo de competencias transferibles a contextos diferentes en el tiempo y en el espacio.

El siglo XxI demanda con urgencia esta adaptación, que en el tema de emprendimien- to debe presentarse en todas las vocaciones y no solo en las típicas licenciaturas de negocios, dado que el emprendimiento y/o autoempleo son características inherentes en muchas disciplinas, como medicina, psicología y derecho, cuya coincidencia es que son profesiones en donde su ejercicio suele darse en un consultorio o despacho en forma independiente.

Para ello, es necesario que el alumno sea una persona activa en el rubro del aprendizaje, considerando que este es un proceso de construcción, a través del cual se adquieren conceptos, principios, procedimientos, valores y actitudes (Zapata, 2015), variables que conjuntamente representan la educación basada en competencias.

En este sentido, el Libro verde de la Comisión de Comunidades Europeas (2003) puntualiza:

El sistema educativo puede aportar tanto competencias como un acercamiento a las empresas que contribuirán a fomentar el espíritu empresarial... En las universidades, la enseñanza del espíritu empresarial no se debería reservar exclusivamente a los alumnos de máster en dirección de empresas, sino ofrecerse también a otros estudiantes (p. 15).

Pero ¿qué significa educar o enseñar emprendimiento? Pertuz-Peralta, Rojas-Caicedo, Navarro-Rodríguez, \& Quintero (2016) refieren al Congreso de Colombia, para plantear que la formación para el emprendimiento busca desarrollar "competencias básicas, competencias laborales, competencias ciudadanas y competencias empresariales dentro del sistema educativo formal y no formal y su articulación con el sector productivo" (p. 32).

Para Simón (2013), la EE es una estrategia para preparar a las nuevas generaciones con un alto grado de creatividad e innovación, cuyo objetivo es desarrollar atributos personales y un conjunto de competencias transversales, así como sistematizar experiencias que permitan desarrollar contenidos en las aulas, además de aptitudes y competencias enfocadas en el saber hacer. 
Según Toca (2010), la EE debe ser holista, enfocada en desarrollar habilidades sin importar su ámbito de desempeño - social, político o público-, cuyo "reto es la formación de individuos capaces de asumir riesgos moderados y calculados, de iniciar proyectos de distinta índole, de propiciar el cambio y el crecimiento de beneficios colectivos" (p. 57).

Siguiendo a Toca (2010), se puede resumir que la $\mathrm{EE}$ forma individuos especializados en tomar decisiones críticas acerca del uso correcto de los recursos (cada día más escasos), para crear y ofrecer valor, y actuando con ética y responsabilidad social.

En México se está empezando a reconocer la importancia de incorporar la $\mathrm{EE}$, según Simón (2013). Como ejemplo, se indica que en 2015 la Secretaría de Educación Pública, a través de la Subsecretaría de Educación Media Superior, desarrolló el Modelo de Emprendedores para la Educación Media Superior (MEEMS) en colaboración con organizaciones de la sociedad civil -Ashoka, Angel Ventures México e Impulsa- y los diferentes subsistemas de la educación media superior.

Por otra parte, para que la EE sea exitosa son precisos dos actores clave: el D y el PE-A. De acuerdo con Pertuz-Peralta et al. (2016), el D formador de emprendedores requiere de características y competencias específicas articuladas de manera integral, para el desarrollo efectivo del PE-A. Estos autores, con base en una amplia revisión del estado del arte, establecen veintidós características/competencias que el $\mathrm{D}$ debe tener.

Seikkula-Leino, Satuvuori, Ruskovaara, \& Hannula (2015) argumentan que en Finlandia la EE, se inicia con la formación del profesorado, es decir, los $\mathrm{D}$ deben prepararse primero en el tema. También los autores señalan que la EE es tanto un método como un contenido de aprendizaje.

Para Robinson, Neergaard, Tanggaard, \& Krueger (2016), el efecto de los programas de educación emprendedora puede depender del método de enseñanza que refleja las creencias del educador; además, recomiendan que la elección del estilo y del método de enseñanza debe relacio- narse según la naturaleza de los alumnos. De ahí que la EE deba centrarse en los estudiantes y en las prácticas de aprendizaje permanente.

Lahn, \& Erikson (2016) presentan un caso con una propuesta que puede encuadrarse como un nuevo modelo de educación para el emprendimiento, el cual busca obtener como resultados de aprendizaje el desarrollo de habilidades de redes sociales, conciencia crítica, sensibilidad intercultural, entre otros. Su propuesta (InvEIR programme) se construyó sobre la base de la economía del conocimiento, que ha generado una nueva ecología, así como una reconfiguración de recursos e instrumentos para la experiencia empresarial que demanda la necesidad urgente de que los estudiantes aprendan a aprender mediante el fomento de su propia conciencia sobre los efectos de diferentes estrategias de acción.

Mandel, \& Noyes (2016) estudiaron la educación para el emprendimiento experiencial con el objetivo de propiciar la actuación de los alumnos en escenarios de incertidumbre y ambigüedad, donde los estu- 
diantes deberían experimentar la complejidad social de interactuar con clientes potenciales o reales y proveedores. Lo más importante de estas experiencias, es la reflexión sobre la realidad en contraste con los escenarios enseñados, imaginados o anticipados. En la educación experiencial del emprendimiento, el proceso es el eje central, a fin de probar la visión y habilidades emprendedoras de los alumnos. El reto es encontrar profesores y mentores adecuados. Trabajar con oportunidades empresariales y confrontar la ambigüedad, así como las ansiedades sociales y profesionales en el mercado son las piedras angulares de este enfoque.

Nielsen, \& Stovang (2015) argumentan que la EE es un proceso de desarrollo de oportunidades, centrado en retos gerenciales y organizacionales que involucran el aprovechamiento de oportunidades en el establecimiento de la marca. Estos autores proponen un modelo denominado DesUni, cuyo enfoque es la generación de ideas y la creación de oportunidades. El modelo utiliza las herramientas de design thinking como medio para guiar a los estudiantes en la búsqueda de oportunidades, a través de un compromiso enfático con los usuarios, el pensamiento divergente y la creación de prototipos.

\section{Metodología}

$\mathrm{n}$ correspondencia con
el propósito descrito, la
presente investigación se sustenta en el paradigma cuantitativo. Se utilizaron métodos teóricos (inductivo-deductivo y analítico-sintético) y estadísticos (descriptivos e inferenciales). El contexto de investigación fue la ciudad de Ensenada, Baja California; en específico, los alumnos de educación superior en el periodo de febrero a marzo de 2017 para realizar un estudio piloto con base en la revisión del estado del arte y considerando la relevancia de la $\mathrm{EE}$, la cual en los últimos años se ha potenciado con apoyo en una búsqueda en Emerald en el periodo de 2011 a 2017, que arrojó 4236 artículos o capítulos y 188 casos de estudio (21\% de los artículos publicados en el último año). Se diseñó una escala para medir la $\mathrm{EE}$, donde los autores base son: Seikkula-Leino, Satu- vuori, Ruskovaara y Hannula (2015); Robinson, Neergaard, Tanggaard y Krueger (2016); y Capella, Gil, Martí y RuizBernardo (2016).

Los ítems diseñados se muestran en la tabla 1 , donde los estudiantes de educación superior valoraron cada ítem, de acuerdo con una escala de Likert, asignando puntajes del 1 al 7, donde 1 significa desacuerdo total y 7 , acuerdo total. La configuración inicial plantea dos factores: el D como promotor de competencias para el emprendimiento (DPCE) y el PE-A. 
Tabla 1. Ítems de la escala EE.

\begin{tabular}{|c|c|}
\hline Código & Ítem \\
\hline DPCE 1 & Los D trabajan con un currículo de competencias. \\
\hline DPCE 2 & $\begin{array}{l}\text { Los D desarrollan la capacidad crítica de los alumnos para entender y manejar los } \\
\text { estándares, y autoevaluarse con criterios y ejemplos explícitos. }\end{array}$ \\
\hline DPCE 3 & $\begin{array}{l}\text { Los D realizan preguntas relevantes que orientan y estimulan la reflexión de los es- } \\
\text { tudiantes. }\end{array}$ \\
\hline DPCE 4 & Los D utilizan estrategias educativas que fomentan la creatividad. \\
\hline DPCE 5 & Los D emplean estrategias educativas que fomentan la innovación. \\
\hline DPCE 6 & Los D promueven la comprensión sobre la memorización. \\
\hline DPCE 7 & Los D utilizan estrategias educativas que fomentan el trabajo en equipo. \\
\hline DPCE 8 & Los D promueven el espíritu empresarial. \\
\hline DPCE 9 & Los D propician la reflexión sobre el uso del dinero. \\
\hline DPCE 10 & Los D promueven el desarrollo de proyectos emprendedores. \\
\hline DPCE 11 & Los D emplean simuladores empresariales. \\
\hline DPCE 12 & $\begin{array}{l}\text { Los D se coordinan para que los proyectos finales sean globalizados e interdiscipli- } \\
\text { nares. }\end{array}$ \\
\hline DPCE 13 & $\begin{array}{l}\text { Los D diseñan actividades de aprendizaje para promover la justicia por medio de la } \\
\text { organización equitativa de tareas, donde cada alumno asume sus responsabilidades } \\
\text { y prioriza el bien común en relación con sus propios intereses. }\end{array}$ \\
\hline PE-A 1 & $\begin{array}{l}\text { El PE-A promueve la reflexión sobre las consecuencias positivas y negativas de los } \\
\text { actos sobre la vida de los demás. }\end{array}$ \\
\hline PE-A 2 & $\begin{array}{l}\text { El PE-A me ha permitido aprender de mis compañeros y aceptar trabajar con perso- } \\
\text { nas diferentes desde una actitud de respeto y colaboración activa. }\end{array}$ \\
\hline PE-A 3 & $\begin{array}{l}\text { El PE-A me ha permitido valorar la rentabilidad de una actividad y cómo sacar bene- } \\
\text { ficio para mejorar y responder a necesidades que se plantean en la vida cotidiana. }\end{array}$ \\
\hline PE-A 4 & $\begin{array}{l}\text { El PE-A me ha ayudado a llevar a cabo ideas creativas con realismo y eficacia, plani- } \\
\text { ficando cómo hacer una cosa detrás de otra y siendo constante a pesar de las dificul- } \\
\text { tades. }\end{array}$ \\
\hline PE-A 5 & $\begin{array}{l}\text { El PE-A me ha permitido reconocer mis aciertos y errores e identificar formas de } \\
\text { hacer mejor las cosas la próxima vez. }\end{array}$ \\
\hline
\end{tabular}

Fuente: elaboración propia. 
Por medio de Google Forms, la escala fue distribuida entre el personal D de tres instituciones de educación superior, para que llevaran a sus alumnos a cumplimentarla. Posteriormente, se realizó el análisis estadístico de los datos, orientado fundamentalmente a la exploración de la estructura factorial, a partir del análisis exploratorio de componentes principales con rotación varimax. Se llevó a cabo el análisis de la confiabilidad, a partir del coeficiente alfa de Cronbach. El procesamiento de los datos se efectuó a través del programa SPSS/Windows 20.0.

El estudio piloto se aplicó a una muestra de sesenta y tres estudiantes. La recomendación clásica, según LloretSegura, Ferreres-Traver, Hernández-Baeza, \& Tomás-Marco (2014), define un tamaño muestral mínimo $(n)$ que oscila entre cincuenta y cuatrocientos sujetos.

\section{ANÁlisis E}

INTERPRETACIÓN DE RESULTADOS

D 1 análisis factorial ex$\checkmark$ ploratorio de ítems es Uuna técnica para validar cuestionarios y explorar el conjunto de variables la- tentes o factores comunes que explican las respuestas a los ítems (Lloret-Segura, FerreresTraver, Hernández-Baeza, \& Tomás-Marco, 2014). También es una técnica que ayuda a determinar las posibles relaciones de interdependencia o correlación entre grupos de variables numéricas medibles, derivadas de conceptos subyacentes representados de variables denominadas factores (Véliz, 2017).

Se aplicó el análisis factorial exploratorio, al no tener criterios que permitieran evaluar la información contenida en los factores encontrados. Entre los requisitos más importantes que debe cumplir la matriz de datos está el que las variables independientes tienen que estar altamente correlacionadas; y, para ello, se tiene que tomar en cuenta el determinante de la matriz de correlaciones. Si este es muy bajo significa que existen variables con intercorrelaciones muy altas, siendo entonces factible continuar con el análisis factorial. Sin embargo, de acuerdo con Montoya (2007), el determinante no debe ser igual a cero, pues en este caso los datos no serían válidos.
Para el caso de este estudio, se obtuvo un determinante igual a $1.856 \mathrm{E}-011$, que al ser muy próximo a cero, indica que el grado de intercorrelación entre las variables es muy alto, por lo que es factible continuar con el análisis factorial (véase tabla 2).

Según Hair, Anderson, Tatham y Black (1999), la prueba de esfericidad de Bartlett contrasta la hipótesis nula de que la matriz de correlaciones observada es, en realidad, una matriz identidad. Al asumir que los datos provienen de una distribución normal multivariante, el estadístico de Bartlett se distribuye, aproximadamente, según el modelo de probabilidad chi-cuadrado y es una transformación del determinante de la matriz de correlaciones. Si el nivel crítico (Sig) es mayor que 0.05 ( $\operatorname{Sig}>0.05)$, no se puede rechazar la hipótesis nula de esfericidad y, en consecuencia, no se puede asegurar que el modelo factorial sea adecuado para los datos. En este caso, el análisis presentó una significancia muy inferior al límite 0.05 , pues fue de 0.000 , lo cual indica que la matriz de datos es válida para continuar con 
Tabla 2. Matriz de correlaciones.

\begin{tabular}{|c|c|c|c|c|c|c|c|c|c|c|c|c|c|c|c|c|c|c|c|}
\hline & & DPCE1 & $\begin{array}{l}\text { DPCE2 } \\
\end{array}$ & DPCE3 & DPCE4 & DPCE5 & DPCE6 & DPCE7 & DPCE8 & DPCE9 & $\begin{array}{l}\text { DPCE10 } \\
\end{array}$ & $\begin{array}{l}\text { DPCE11 } \\
\end{array}$ & DPCE12 & $\overline{D P C E} 13$ & $\begin{array}{l}E-A 1 \\
\end{array}$ & PE-A2 & PE-A3 & PE-A4 & PE-A5 \\
\hline \multirow[t]{18}{*}{ 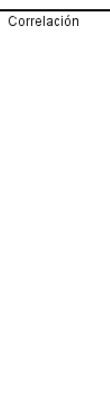 } & DPCE1 & 1.000 & .632 & .658 & .573 & .543 & .576 & .630 & .524 & .481 & 509 & $\begin{array}{r}476 \\
\end{array}$ & \begin{tabular}{|r|}
.481 \\
\end{tabular} & .480 & .603 & $\frac{651}{6}$ & .523 & .539 & .323 \\
\hline & DPCE2 & .632 & 1.000 & .767 & .808 & .794 & .709 & .714 & 659 & .543 & 601 & .494 & .567 & .572 & .646 & .680 & .597 & .731 & .483 \\
\hline & DPCE3 & 658 & .767 & 1.000 & .743 & 689 & 697 & .705 & .657 & .559 & .653 & .595 & .599 & .644 & .694 & .784 & .673 & .718 & .698 \\
\hline & DPCE4 & .573 & .808 & .743 & 1.000 & .913 & .683 & .800 & .714 & .648 & .683 & .632 & .678 & .712 & .745 & .741 & .712 & .818 & .703 \\
\hline & $\begin{array}{l}\text { DPCE5 } \\
\text { DP }\end{array}$ & .543 & .794 & .689 & .913 & 1.000 & .722 & .728 & .737 & .709 & .701 & .643 & .701 & .708 & .732 & .671 & .744 & .818 & .678 \\
\hline & DPCE6 & .576 & .709 & 697 & .683 & .722 & 1.000 & .625 & .649 & .586 & .634 & .545 & .595 & .577 & .667 & .664 & .727 & .744 & .577 \\
\hline & DPCE7 & .630 & .714 & .705 & .800 & .728 & .625 & 1.000 & .619 & .557 & .553 & .496 & .560 & .666 & .650 & .649 & .590 & .705 & .639 \\
\hline & DPCE8 & .524 & .659 & .657 & .714 & .737 & 649 & .619 & 1.000 & .845 & .804 & .756 & .767 & .700 & .674 & .758 & .750 & .766 & .581 \\
\hline & DPCE9 & .481 & .543 & .559 & 648 & .709 & .586 & .557 & .845 & 1.000 & .830 & .820 & .799 & .720 & .686 & .714 & .740 & .702 & .553 \\
\hline & DPCE10 & .509 & 601 & .653 & .683 & .701 & .634 & .553 & .804 & .830 & 1.000 & .787 & .809 & .735 & .682 & .758 & .764 & .805 & .618 \\
\hline & DPCE11 & .476 & .494 & .595 & .632 & .643 & .545 & .496 & .756 & .820 & .787 & 1.000 & .901 & .734 & .673 & .621 & .634 & 601 & .552 \\
\hline & DPCE12 & .481 & .567 & .599 & .678 & .701 & .595 & .560 & .767 & .799 & .809 & .901 & 1.000 & .824 & .763 & .645 & .708 & .725 & .634 \\
\hline & DPCE13 & .480 & .572 & 644 & .712 & .708 & .577 & .666 & .700 & .720 & .735 & .734 & .824 & 1.000 & .815 & .714 & .753 & .746 & .734 \\
\hline & PE-A1 & .603 & .646 & .694 & .745 & .732 & .667 & .650 & .674 & .686 & .682 & .673 & .763 & .815 & 1.000 & .838 & .845 & .777 & .686 \\
\hline & & 651 & .680 & .784 & .741 & .671 & .664 & .649 & .758 & .714 & .758 & .621 & 645 & .714 & .838 & 1.000 & .831 & .777 & .661 \\
\hline & PE-A3 & .523 & .597 & .673 & .712 & .744 & .727 & .590 & .750 & .740 & .764 & .634 & .708 & .753 & .845 & .831 & 1.000 & .847 & .781 \\
\hline & PE-A4 & .539 & .731 & .718 & .818 & .818 & .744 & .705 & .766 & .702 & .805 & .601 & .725 & .746 & .777 & .777 & .847 & 1.000 & .728 \\
\hline & $\mathrm{PE}$ & .323 & .483 & 698 & .703 & 678 & .577 & .639 & .581 & .553 & .618 & .552 & .634 & .734 & .686 & .661 & .781 & .728 & 1.000 \\
\hline \multirow[t]{18}{*}{ Sig. (Unilateral) } & DPCE1 & & .000 & .000 & .000 & .000 & .000 & .000 & .000 & .000 & .000 & .000 & .000 & .000 & .000 & .000 & .000 & .000 & .009 \\
\hline & DPCE2 & .000 & & .000 & .000 & .000 & .000 & .000 & .000 & .000 & .000 & .000 & .000 & .000 & .000 & .000 & .000 & .000 & .000 \\
\hline & DPCE3 & .000 & .000 & & .000 & .000 & .000 & .000 & .000 & .000 & .000 & .000 & .000 & .000 & .000 & .000 & .000 & .000 & .000 \\
\hline & DPCE4 & .000 & .000 & .000 & & .000 & .000 & .000 & .000 & .000 & .000 & .000 & .000 & .000 & .000 & .000 & .000 & .000 & .000 \\
\hline & DPCE5 & .000 & .000 & .000 & .000 & & .000 & .000 & .000 & .000 & .000 & .000 & .000 & .000 & .000 & .000 & .000 & .000 & .000 \\
\hline & $\begin{array}{l}\text { DPCE6 } \\
\text { DP }\end{array}$ & .000 & .000 & .000 & .000 & .000 & & .000 & .000 & .000 & .000 & .000 & .000 & .000 & .000 & .000 & .000 & .000 & .000 \\
\hline & DPCE7 & .000 & .000 & .000 & .000 & .000 & .000 & & .000 & .000 & .000 & .000 & .000 & .000 & .000 & .000 & .000 & .000 & .000 \\
\hline & DPCE8 & .000 & .000 & .000 & .000 & .000 & .000 & .000 & & .000 & .000 & .000 & .000 & .000 & .000 & .000 & .000 & .000 & .000 \\
\hline & DPCE9 & .000 & .000 & .000 & .000 & .000 & .000 & .000 & .000 & & .000 & .000 & .000 & .000 & .000 & .000 & .000 & .000 & .000 \\
\hline & DPCE10 & .000 & .000 & .000 & .000 & .000 & .000 & .000 & .000 & .000 & & .000 & .000 & .000 & .000 & .000 & .000 & .000 & .000 \\
\hline & DPCE11 & .000 & .000 & .000 & .000 & .000 & .000 & .000 & .000 & .000 & .000 & & .000 & .000 & .000 & .000 & .000 & .000 & .000 \\
\hline & DPCE 12 & .000 & .000 & .000 & .000 & .000 & .000 & .000 & .000 & .000 & .000 & .000 & & .000 & .000 & .000 & .000 & .000 & .000 \\
\hline & DPCE13 & .000 & .000 & .000 & .000 & .000 & .000 & .000 & .000 & .000 & .000 & .000 & .000 & & .000 & .000 & .000 & .000 & .000 \\
\hline & PE-A1 & .000 & .000 & .000 & .000 & .000 & .000 & .000 & .000 & .000 & .000 & .000 & .000 & .000 & & .000 & .000 & .000 & .000 \\
\hline & PE-A2 & .000 & .000 & .000 & .000 & .000 & .000 & .000 & .000 & .000 & .000 & .000 & .000 & .000 & .000 & & .000 & .000 & .000 \\
\hline & PE-A3 & .000 & .000 & .000 & .000 & .000 & .000 & .000 & .000 & .000 & .000 & .000 & .000 & .000 & .000 & .000 & & .000 & .000 \\
\hline & PE-A4 & .000 & .000 & .000 & .000 & .000 & .000 & .000 & .000 & .000 & .000 & .000 & .000 & .000 & .000 & .000 & .000 & & .000 \\
\hline & PE-A5 & .009 & .000 & .000 & .000 & .000 & .000 & .000 & .000 & .000 & .000 & .000 & .000 & .000 & .000 & .000 & .000 & .000 & \\
\hline
\end{tabular}

Fuente: elaboración propia, a partir de cálculos con el programa spss.

el proceso de análisis factorial (véase tabla 3).

Los valores del Índice de Kaiser-Meyer-Olkin (KMO) entre 0.5 y 1 , indican que es apropiado aplicar el análisis factorial a la matriz de datos en estudio (Montoya, 2007). En el caso de la matriz de datos que se analiza, se obtuvo un Índice de KMO de 0.920, lo que indica que se acepta la conveniencia de la aplicación, es decir, la muestra tomada para el estudio es apropiada y, por lo tanto, se puede con- tinuar con la aplicación del análisis factorial (Véliz, 2017).

El siguiente elemento a analizar es la matriz de varianzas-covarianzas antiimagen y la matriz de correlaciones antiimagen. Para Véliz (2017), Montoya (2007) y Hair et al. (1999), la matriz de correlaciones antiimagen se encuentra relacionada con la inversa de la matriz de correlaciones y se utiliza como diagnóstico de la adecuación de los datos a un modelo factorial. En este contexto, un coeficiente de corre- lación parcial expresa el grado de relación entre dos variables, tras eliminar el efecto de las restantes variables incluidas en el análisis.

Cuando las variables incluidas en el análisis comparten una gran cantidad de información, debido a la presencia de factores comunes, la correlación parcial entre cualquier par de variables debe ser reducida. Por el contrario, cuando dos variables comparten una gran cantidad de información entre ellas, pero no con el resto 
de las demás variables (en consecuencia, tampoco con los factores comunes), la correlación parcial entre ellas será elevada, siendo esto un mal síntoma de cara a la idoneidad del análisis factorial. Por otra parte, las correlaciones parciales son también estimaciones de las correlaciones entre

Tabla 3. Índice de KMO y prueba de Bartlett.

\begin{tabular}{|c|c|c|}
\hline \multicolumn{2}{|c|}{$\begin{array}{l}\text { Medida de adecuación muestral de Kaiser-Meyer- } \\
\text { Olkin. }\end{array}$} & .920 \\
\hline $\begin{array}{l}\text { Prueba de esfericidad de } \\
\text { Bartlett }\end{array}$ & $\begin{array}{l}\text { Chi-cuadrado } \\
\text { aproximado }\end{array}$ & 1116.063 \\
\hline & $\mathrm{gl}$ & 153 \\
\hline & Sig. & .000 \\
\hline
\end{tabular}

Fuente: elaboración propia, a partir de cálculos con el programa SPSS. los factores únicos (existe un valor único para cada variable del modelo).

En este caso, los coeficientes tienden al cero, lo cual representa un excelente indicador con respecto a la bondad o pertinencia de que se puede continuar con el análisis factorial (véase tabla 4). Los valores de la diagonal de la matriz de covarianza antiimagen, se obtienen restando a 1 la correlación múltiple al cuadrado entre cada variable y las restantes variables del análisis; por lo tanto, representan una esti-

Tabla 4. Matriz antiimagen.

\begin{tabular}{|c|c|c|c|c|c|c|c|c|c|c|c|c|c|c|c|c|c|c|c|}
\hline \\
\hline \multirow{19}{*}{ Covarianza anti-imagen } & & DPCE1 & DPCE2 & DPCE3 & DPCE4 & DPCE5 & DPCE6 6 & DPCE7 & DPCE8 & DPCE9 & \begin{tabular}{|l|l|} 
DPCE10 \\
\end{tabular} & DPCE11 & DPCE12 & DPCE13 & PEE-A1 & PE-A2 & PE-A3 & PE-A4 & PE-A5 \\
\hline & DPCE1 & .345 & .008 & $\begin{array}{l}-0.067 \\
\end{array}$ & .012 & -016 & $\begin{array}{r}.018 \\
\end{array}$ & -109 & .025 & .020 & $\begin{array}{l}.004 \\
\end{array}$ & & & & .006 & -033 & .030 & .016 & .100 \\
\hline & DPCE2 & .008 & .172 & -.074 & -.023 & . & - .037 & -.021 & -.017 & . 013 & -.002 & & -.024 & .002 & .002 & -.008 & .004 & -.003 & \\
\hline & DPCE3 & -.067 & -.074 & .164 & .015 & .005 & . .031 & .001 & .006 & .024 & -.003 & .039 & .024 & .005 & .001 & -.037 & .030 & .010 & -.083 \\
\hline & DPCE4 & .012 & -.023 & .015 & .088 & . 059 & .023 & .044 & .005 & .025 & .009 & .026 & .006 & .008 & .001 & -.032 & .017 & . .022 & .018 \\
\hline & DPCE5 & -016 & .034 & .005 & .059 & .096 & .025 & .021 & .010 & - .036 & .004 & .006 & .008 & . .004 & .014 & .042 & .012 & . .002 & -.013 \\
\hline & DPCEE & -.018 & -.037 & -031 & .023 & .025 & .312 & -.031 & .002 & .014 & .004 & -.016 & $3.863 \mathrm{E}-005$ & .030 & .001 & .010 & .044 & -025 & .014 \\
\hline & DPCE7 & -109 & -.021 & .001 & .044 & .021 & - .031 & .231 & -.023 & - .039 & .020 & .019 & & -.046 & -.009 & .017 & .039 & -.017 & .057 \\
\hline & DPCE8 & .025 & -017 & -.006 & . 005 & -.010 & .002 & -.023 & .177 & -.049 & .011 & -.015 & -.018 & -.001 & .043 & .036 & .020 & -.019 & .024 \\
\hline & DPCE9 & .020 & .013 & .024 & .025 & -.036 & .014 & .039 & .049 & .148 & -.031 & .038 & .000 & -.004 & . 007 & .025 & .017 & .008 & .021 \\
\hline & DPCE10 & -004 & .002 & -.003 & .009 & -.004 & .004 & .020 & .011 & - .031 & .147 & -025 & -019 & -.015 & .045 & -037 & -.005 & -.054 & .006 \\
\hline & DPCE11 & -.009 & .033 & -.039 & . .026 & .006 & -.016 & .019 & -.015 & -.038 & -.025 & .106 & -.060 & $8.827 \mathrm{E}-006$ & -.003 & .012 & .001 & .043 & .011 \\
\hline & DPCE12 & -.014 & .024 & .024 & .006 & .008 & 3.863E-005 & .013 & .018 & .000 & .019 & .060 & .091 & -032 & -.035 & .026 & .014 & -.023 & .024 \\
\hline & DPCE13 & .014 & .002 & .005 & .008 & -.004 & .030 & -.046 & .001 & .004 & .015 & 8.827E-006 & .032 & .190 & .043 & .001 & .005 & . .001 & .034 \\
\hline & PE-A1 & -.006 & .002 & .001 & .001 & -0.014 & .001 & . .009 & .043 & .007 & .045 & .003 & .035 & .043 & .117 & -.053 & . .038 & -.006 & .025 \\
\hline & PE-A2 & -033 & -.008 & - .037 & - .032 & .042 & .010 & .017 & .036 & .025 & - .037 & .012 & .026 & -001 & -.053 & .107 & . .021 & .010 & -.002 \\
\hline & PE-A3 & -.030 & .004 & .030 & .017 & .012 & -. .044 & .039 & .020 & -017 & -.005 & .001 & .014 & .005 & -.038 & -.021 & .099 & . .031 & -.062 \\
\hline & PE-A4 & .016 & -.003 & -.010 & -.022 & -.002 & -.025 & -.017 & -.019 & .008 & -.054 & .043 & -023 & -001 & -.006 & .010 & - .031 & .119 & .003 \\
\hline & PE-A5 & .100 & .065 & .083 & .018 & -.013 & .014 & .057 & .024 & . 021 & .006 & .011 & . .024 & -.034 & .025 & . .002 & .062 & .003 & .161 \\
\hline \multirow[t]{18}{*}{ Correlación anti-imagen } & DPCE1 & $.908^{\mathrm{a}}$ & .033 & -.282 & .067 & .087 .087 & .054 & .386 & .101 & .088 & .018 & .047 & -080 & .056 & .032 & -171 & -161 & .077 & .425 \\
\hline & DPCE2 & .033 & $.923^{\mathrm{a}}$ & -.440 & -186 & -.262 & -160 & -104 & -095 & . 081 & -.011 & .246 & -195 & .009 & .012 & -.059 & .028 & -.024 & .393 \\
\hline & DPCE3 & -.282 & -.440 & $.902^{\mathrm{a}}$ & .127 & .041 & -139 .139 & .005 & -.035 & .153 & -.017 & -299 & .192 & .029 & .007 & -.278 & .233 & -.075 & -.511 \\
\hline & & .067 & -.186 & .127 & $.906^{\mathrm{a}}$ & -.642 & .138 &. .311 & .043 & .219 & .076 & -.272 & .072 & .064 & .013 & .335 & .180 & .213 & -.152 \\
\hline & DPCE5 & .087 & .262 & .041 & .642 & $.912^{\mathrm{a}}$ & .143 & .143 & .078 & .299 & . .034 & .056 & .082 & . .031 & . .136 & .419 & .122 & .022 & .108 \\
\hline & DPCE6 & -.054 & -160 & 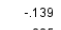 & .138 & -143 & $.971^{\mathrm{a}}$ & -.115 & .007 & .066 & .017 & .090 & .000 & .122 & .007 & .055 & -253 & -131 & .062 \\
\hline & DPCE7 & -.386 & -104 & .005 & -.311 & .143 & -.115 & $.921^{\mathrm{a}}$ & -.113 & -.211 & .106 & .124 & .090 & -.217 & -057 & .107 & .260 & -103 & -295 \\
\hline & DPCE8 & .101 & .095 & .035 & .043 & .078 & .007 & -.113 & $.956^{\mathrm{a}}$ & .306 & .070 & -.113 & -.144 & -.005 & .302 & -.262 & -153 & -.130 & .141 \\
\hline & & .088 & .081 & .153 & .219 & .299 & .066 & .211 & .306 & $.937^{\mathrm{a}}$ & .213 & .304 & .001 & .021 & .054 & .197 & -143 & . 057 & .136 \\
\hline & DPCE10 & -.018 & -011 & -017 & .076 & . 034 & .017 & .106 & .070 & .213 & $.942^{\mathrm{a}}$ & -.202 & -163 & .091 & .346 & .298 & .044 & .410 & .042 \\
\hline & DPCE11 & -.047 & .246 & -299 & -.272 & .056 & - .090 & .124 & - -113 & -.304 & -202 & $.886^{\mathrm{a}}$ & -611 & $6.207 \mathrm{E}-005$ & -.029 & .110 & .006 & .386 & .088 \\
\hline & CCE12 & -.080 & -195 & .192 & .072 & .082 & .000 & .090 & -144 & .001 & -163 & -611 & $.905^{\mathrm{a}}$ & -.242 & -.335 & .263 & .150 & -.221 & -198 \\
\hline & DPCE13 & .056 & .009 & .029 & .064 & -031 & .122 & .217 & .005 & -021 & . 091 & 6.207E-005 & -.242 & $.970^{\mathrm{a}}$ & . .286 & -.009 & .038 & -.007 & -197 \\
\hline & PE-A1 & -.032 & .012 & .007 & .013 & -136 & .007 & .057 & .302 & .054 & .346 & $\begin{array}{r}-.029 \\
\end{array}$ & .335 & -.286 & $.915^{\mathrm{a}}$ & .470 & .358 & -.054 & .183 \\
\hline & PE-A2 & - 171 & -.059 & -.278 & -.335 & .419 & .055 & .107 & .262 & -197 & - 2998 & .110 & .263 & - .009 & - -.470 & $.901^{\mathrm{a}}$ & .202 & .087 & -.013 \\
\hline & PE-A3 & -161 & .028 & .233 & .180 & -.122 & -.253 & .260 & -153 & -143 & -.044 & .006 & .150 & .038 & -.358 & .202 & $.915^{\mathrm{a}}$ & -290 & -497 \\
\hline & PE-A4 & .077 & -.024 & . 075 & -.213 & -.022 & . 131 & -103 & .130 & .057 & .410 & .386 & .221 & -.007 & .054 & .087 & . .290 & $.943^{\mathrm{a}}$ & .025 \\
\hline & PE-A5 & .425 & .393 & -.511 & -.152 & . .108 & .062 & .295 & .141 & .136 & .042 & . 088 & -198 & .197 & .183 & .0.013 & -.497 & .025 & $.858^{\mathrm{a}}$ \\
\hline
\end{tabular}

Fuente: elaboración propia, a partir de cálculos con el programa sPss. 
Figura 3. Gráfico de sedimentación.

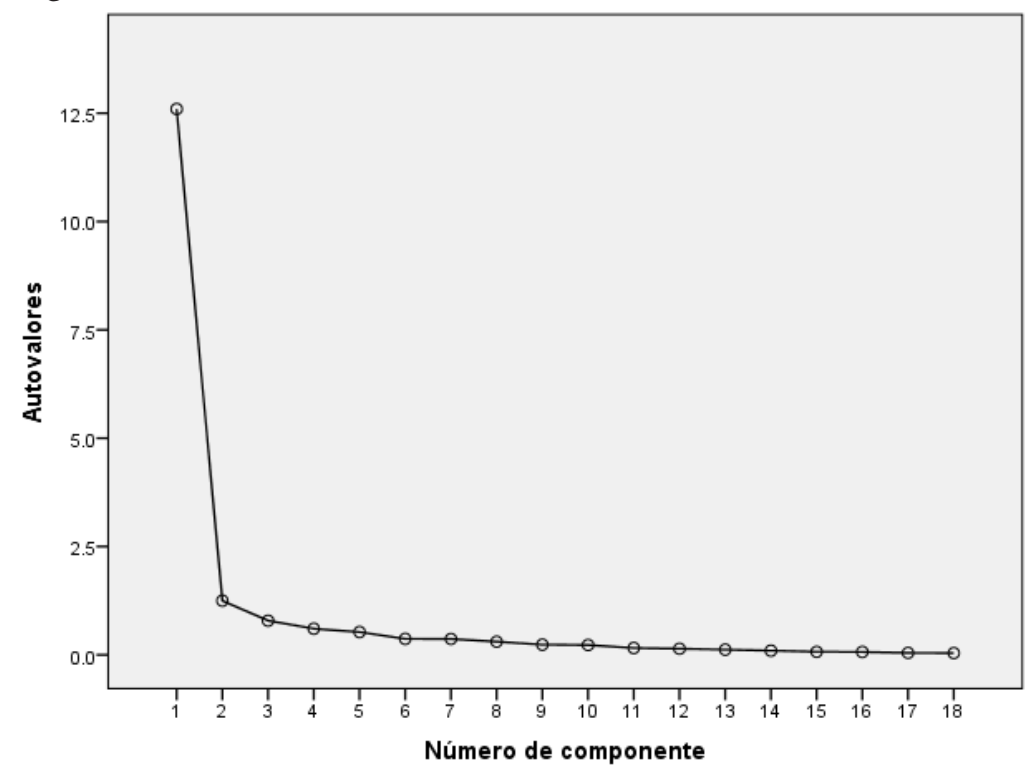

Fuente: elaboración propia, a partir de cálculos con el programa sPSs (año).

mación de la unicidad de cada variable o, lo que es lo mismo, una estimación de lo que cada variable tiene de propio o de no compartido con las demás variables. Es una herramienta para comprobar la factibilidad de la aplicación del análisis factorial. En el caso de la matriz de correlación antiimagen que estamos trabajando, todos los valores de la diagonal presentan cifras altas cercanas a 1 . Por lo tanto, estos resultados proporcionan otro indicador positivo sobre la matriz de da- tos que da luz verde al análisis factorial.

Esta primera etapa del análisis factorial, permite comprobar satisfactoriamente todos los tipos de análisis sobre la pertinencia y validez de la matriz de datos. Con ello, se procede a desarrollar la segunda etapa que consiste en la extracción de los distintos factores, a través de la agrupación de los dieciocho ítems originales en nuevas variables que se denominan componentes o factores, que son combinaciones de las variables originales.
Tabla 5. Comunalidades.

\begin{tabular}{|l|c|r|}
\hline & Inicial & Extracción \\
\hline DPCE1 & 1.000 & .579 \\
DPCE2 & 1.000 & .799 \\
DPCE3 & 1.000 & .772 \\
DPCE4 & 1.000 & .832 \\
DPCE5 & 1.000 & .794 \\
DPCE6 & 1.000 & .680 \\
DPCE7 & 1.000 & .741 \\
DPCE8 & 1.000 & .781 \\
DPCE9 & 1.000 & .831 \\
DPCE10 & 1.000 & .822 \\
DPCE11 & 1.000 & .823 \\
DPCE12 & 1.000 & .867 \\
DPCE13 & 1.000 & .773 \\
PE-A1 & 1.000 & .771 \\
PE-A2 & 1.000 & .771 \\
PE-A3 & 1.000 & .785 \\
PE-A4 & 1.000 & .821 \\
PE-A5 & 1.000 & .602 \\
\hline \multicolumn{2}{|c|}{ Método de extracción: Análisis de } \\
Componentes principales.
\end{tabular}

Fuente: elaboración propia, a partir de cálculos con el programa sPSs (año).

Según Véliz (2017), las comunalidades miden la cantidad de información que los factores comunes expresan de cada variable e indican iniciales que valen 1 , porque se ha elegido el método de componentes principales. El gráfico de sedimentación indica que solo son mayores que 1 , los autovalores de las dos primeras variables, con lo que estas dos 
Tabla 6. Varianza total explicada.

\begin{tabular}{|c|c|c|c|c|c|c|}
\hline \multirow[b]{2}{*}{ Componente } & \multicolumn{3}{|c|}{ Autovalores iniciales } & \multicolumn{3}{|c|}{$\begin{array}{l}\text { Sumas de las saturaciones al cuadrado de la } \\
\text { extracción }\end{array}$} \\
\hline & Total & $\begin{array}{l}\% \text { de la } \\
\text { varianza }\end{array}$ & $\%$ acumulado & Total & $\begin{array}{l}\% \text { de la } \\
\text { varianza }\end{array}$ & $\%$ acumulado \\
\hline 1 & 12.596 & 69.980 & 69.980 & 12.596 & 69.980 & 69.980 \\
\hline 2 & 1.249 & 6.939 & 76.919 & 1.249 & 6.939 & 76.919 \\
\hline 3 & .788 & 4.378 & 81.298 & & & \\
\hline 4 & .603 & 3.352 & 84.650 & & & \\
\hline 5 & .527 & 2.925 & 87.575 & & & \\
\hline 6 & .369 & 2.050 & 89.625 & & & \\
\hline 7 & .367 & 2.038 & 91.663 & & & \\
\hline 8 & .303 & 1.686 & 93.349 & & & \\
\hline 9 & .235 & 1.306 & 94.655 & & & \\
\hline 10 & .226 & 1.256 & 95.911 & & & \\
\hline 11 & .158 & .878 & 96.789 & & & \\
\hline 12 & .141 & .781 & 97.570 & & & \\
\hline 13 & .120 & .666 & 98.235 & & & \\
\hline 14 & .095 & .529 & 98.764 & & & \\
\hline 15 & .071 & .394 & 99.158 & & & \\
\hline 16 & .066 & .369 & 99.526 & & & \\
\hline 17 & .045 & .249 & 99.775 & & & \\
\hline 18 & .040 & .225 & 100.000 & & & \\
\hline
\end{tabular}

Método de extracción: Análisis de Componentes principales.

Fuente: elaboración propia, a partir de cálculos con el programa sPSs (đй̃o).

variables resumirán al resto, representándolas de forma coherente, es decir, serán los dos componentes principales que resumen toda la información.

La varianza total explicada ilustra detalladamente la selección de los dos componentes principales. Como puede verse en la tabla 6 , únicamente los dos primeros factores tienen valores propios mayores que 1 y explican el $76.919 \%$ de la varianza, es decir, solo son relevantes dos factores para resumir las variables originales de la escala.

La matriz de componentes (véase tabla 7), con el método de extracción de análisis de componentes principales, recoge la carga o ponderación de cada factor en cada una de las variables, es decir, la correlación existente entre cada variable y cada factor. Las cargas altas indican que esa variable es representativa para el mencionado factor. Por ejemplo, podemos ver que la variable DPCE 1 (Los D trabajan con un currículo de competencias) es atribuible al factor 1 , 
Figura 4. Gráfico de componentes

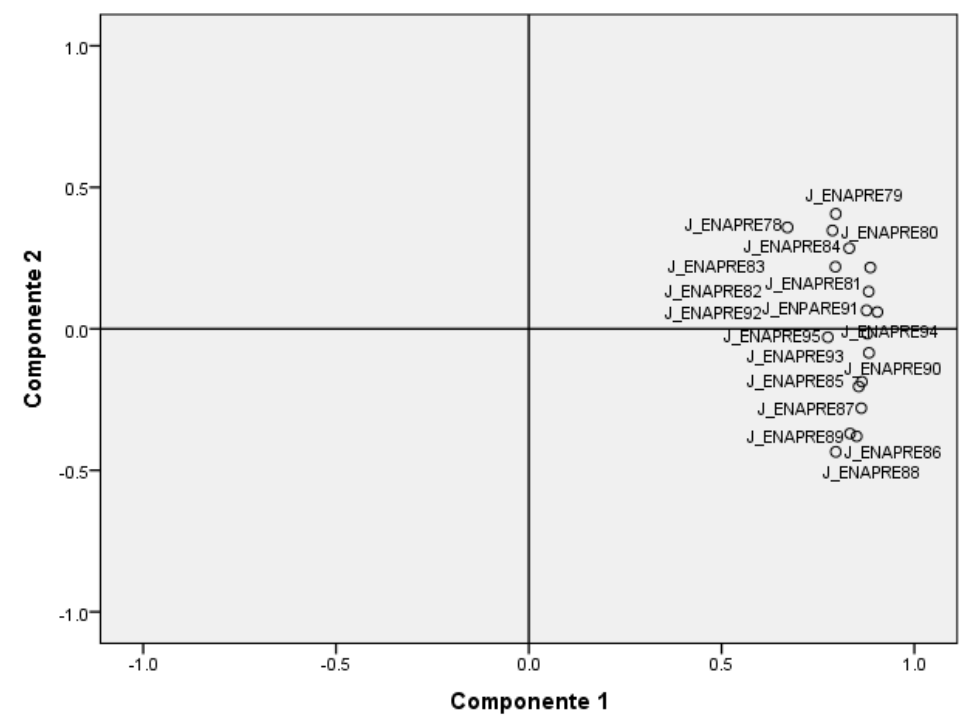

Fuente: elaboración propia, a partir de cálculos con el programa SPSS.

debido a que es en él donde se tiene una mayor carga (0.671). En este caso, todas las variables son atribuibles al factor 1 ; además, se cumple con la condición de que cada variable, se cargue solo sobre un factor mayor de 0.5 y cercano a $1-\mathrm{y}$ el resto hacia valores cercanos a 0. Por lo tanto, el factor $1 \mathrm{se}$ compone de las dieciocho variables (componente general) y considerando que las cargas son claras, se infiere que no existe ambigüedad en la selección de las variables por cada factor (véase figura 4).
No obstante la claridad en la carga factorial de las variables mostrada por la matriz de carga de factores (véase tabla 7), resulta necesario efectuar una rotación ortogonal que permitirá reducir ambigüedades en las cargas factoriales de las variables y hallar una solución más clara. En la práctica, el objetivo de los métodos de rotación es simplificar filas o columnas de la matriz de factores para facilitar la interpretación.

El método de rotación utilizado es varimax, el cual busca redistribuir la varianza a lo largo de todos los componen-
Tabla 7. Matriz de componentes.

\begin{tabular}{|l|l|r|}
\hline \multirow{2}{*}{} & \multicolumn{2}{|l|}{ Componente } \\
\cline { 2 - 3 } & 1 & \multicolumn{1}{l|}{2} \\
\hline DPCE1 & .671 & .358 \\
DPCE2 & .796 & .406 \\
DPCE3 & .831 & .285 \\
DPCE4 & .886 & .217 \\
DPCE5 & .882 & .132 \\
DPCE6 & .795 & .219 \\
DPCE7 & .787 & .347 \\
DPCE8 & .864 & -.187 \\
DPCE9 & .833 & -.370 \\
DPCE10 & .862 & -.280 \\
DPCE11 & .796 & -.435 \\
DPCE12 & .850 & -.380 \\
DPCE13 & .856 & -.204 \\
PE-A1 & .878 & -.017 \\
PE-A2 & .876 & .065 \\
PE-A3 & .882 & -.085 \\
PE-A4 & .904 & .059 \\
PE-A5 & .776 & -.030 \\
\hline
\end{tabular}

Método de extracción: Análisis de componentes principales.

$$
\begin{aligned}
& \text { a. } 2 \text { componentes } \\
& \text { extraídos }
\end{aligned}
$$

Fuente: elaboración propia, a partir de cálculos con el programa SPSs (año).

tes en la matriz de carga. Con ello, se simplifica el modelo y se obtienen resultados más claros para identificar los factores en cada componente, pues este método aproxima las cargas altas a $10-1$ y las cargas bajas de la matriz no rotada, a 0 , eliminando, de esta forma, las ambigüedades existentes en 
la matriz no rotada. Con esta rotación, se obtienen nuevos valores y nuevos vectores propios, así como diferentes porcentajes de explicación, pero se mantiene la variación total de los dos componentes, que es $76.919 \%$ (véase tabla 8 ). Sin embargo, se observa que los ítems se distribuyen, según sus cargas, en forma distinta a como se han observado en la matriz sin rotar.

En el factor 1, se agruparon nueve ítems relacionados con la percepción acerca de la actuación del $\mathrm{D}$ promotor de competencias para el emprendimiento, que explicó $69.98 \%$ de la varianza. En el factor 2 , se agruparon nueve ítems correspondientes al PE-A, que explicó $6.93 \%$ de la varianza.

Por último, se valora la fiabilidad global de la escala de EE, cuyo resultado del alfa de Cronbach es 0.974 , un valor cercano a 1 que se considera muy bueno. También se valoró la confiabilidad por separado para las dimensiones; en el caso de la dimensión $\mathrm{D}$, el resultado fue de 0.962 , mientras que para el PE-A fue de 0.945, ambos muy cercanos a 1 , lo cual implica que la escala es confiable en su medición.

Tabla 8. Matriz de componentes rotados.

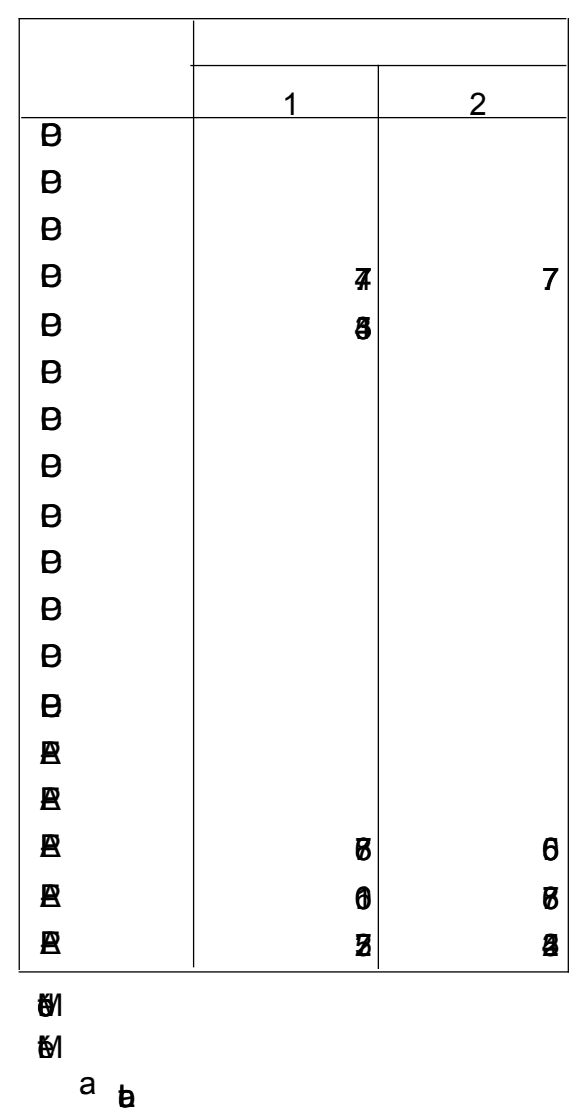

Fuente: elaboración propia, a partir de cálculos con el programa SPSs.

\section{Conclusiones}

a escala de EE satisface
los criterios de validez
y fiabilidad exigibles en un instrumento científico. El procedimiento de validez del instrumento, se inició desde su construcción en donde se siguió un proceso estandari- zado, riguroso, objetivo y basado en criterios de calidad. El constructo EE se definió en términos operativos y tras el estudio de campo, se realizó un análisis factorial, que reveló la existencia de dos factores diferentes con propiedades psicométricas adecuadas. 
La escala de EE presenta una elevada consistencia interna, superior a 0.9 en todos los factores, por lo que se puede afirmar que los ítems están agrupados en forma homogénea en cada uno de los factores. Además, se indica que el test mide un constructo coherente y riguroso, puesto que el coeficiente global también resultó ser significativo.

Se recomienda probar esta escala con una muestra más amplia y también se sugiere validarla con adultos y adolescentes para estandarizar el instrumento. En la misma medida, se podría mejorar el estudio de las propiedades psicométricas de este instrumento, introduciendo medidas de fiabilidad test-retest, sensibilidad del instrumento a efectos del tratamiento o planteando estudios longitudinales.

\section{REFERENCIAS}

\section{BIBLIOGRÁFICAS}

Buendía-Martínez, I., \& Carrasco, I. (2013). Mujer, actividad emprendedora y desarrollo rural en América Latina y el Caribe. Cuadernos de Desarrollo Rural, 10(72), 21-45.

Campos Vázquez, R. M. (2015). El salario mínimo y el empleo: eviden- cia internacional y posibles impactos para el caso mexicano. Economía UNAM, 12(36), 90-106. Recuperado de http:// www.redalyc.org/articulo. oa? id=363542904006

Capella Peris, C., Gil Gómez, J., Martí Puig, M., \& Ruiz-Bernardo, P. (2016). Construcción de un cuestionario para medir el emprendimiento social en educación física. Pedagogia Social. Revista Interuniversitaria, 28, 169-188. Recuperado de http:// www.redalyc.org/articulo. oa? id $=135047100014$

Comisión de Comunidades Europeas (2003). Libro verde: el espiritu empresarial en Europa. Recuperado de www.oei.es/historico/ etp/green_paper_final_ es.pdf

Flores Payán, L., \& Salas Durazo, I. A. (2015). Las brechas de género en la calidad del empleo en México. Una valoración basada en modelos de lógica difusa. Revista Análisis Económico, $X X X(75), 89-112$.

Hair, Jr., J. F., Anderson, R. E., Tatham, R. L., \& Black, W. C. (1999). Análisis multi- variante. Madrid: Prentice Hall Iberia.

Instituto Nacional de Estadística y Geografía (Inegi) (2017). Remuneraciones. Salarios en la industria manufacturera. Países seleccionados. Recuperado de http://www.beta.inegi. org.mx/datos/

Lahn, L. C., \& Erikson, T. (2016). Entrepreneurship Education by Design. Education + Training, 58(7/8), 684-699.

Lloret-Segura, S., FerreresTraver, A., Hernández-Baeza, A., \& Tomás-Marco, I. (2014). El análisis factorial exploratorio de los ítems: una guía práctica, revisada y actualizada. Anales de Psicología, 30(3), 1151-1169. Recuperado de https:// dx.doi.org/10.6018/analesps.30.3.199361

Mandel, R., \& Noyes, E. (2016). Survey of Experiential Entrepreneurship Education Offerings among Top Undergraduate Entrepreneurship Programs. Education + Training, 58(2), 164-178.

Montoya Suárez, O. (2007). Aplicación del análisis factorial a la investigación de mercados: caso de es- 
tudio. Scientia et Technica, $\operatorname{XIII}(35), \quad 281-286$. Recuperado de http:// www.redalyc.org/articulo. oa $?$ id $=84903549$

Munuera, M. P. y Gómez, F. (2016) Innovación y emprendimiento social en el proceso de enseñanza: nuevas narrativas universitarias. Opción, 32 (8). pp. 329-348. http://eprints. ucm.es/39936/

Nielsen, S. L., \& Stovang, P. (2015). DesUni: University Entrepreneurship Education through Design Thinking. Education + Training, 57(8/9), 977 991.

Organización de las Naciones Unidas para la Educación, la Ciencia y la Cultura (Unesco) (1998). Declaración mundial sobre la educación superior en el siglo XXI: visión y acción. Recuperado de http://www. unesco.org/education/ educprog/wche/declaration_spa.htm

Pertuz-Peralta, V. P., RojasCaicedo, G. Y., NavarroRodríguez, A., \& Quintero, L. T. (2016). Perfil docente y fomento de la cultura del emprendimiento: búsqueda de una rela- ción. Educación y Educadores, 19(1), 29-45.

Restrepo Salgado, E., Tapasco Triviño, Ó. J., \& Vidarte Claros, J. A. (2016). Perfil emprendedor de estudiantes de la tecnología en gestión agropecuaria, Universidad de Caldas. Anfora, 23(41), 87-106.

Robinson, S., Neergaard, H., Tanggaard, L., \& Krueger, N. F. (2016). New Horizons in Entrepreneurship Education: from Teacherled to Student-centered Learning. Education + Training, 58(7/8), 661-683.

Sanabria-Rangel, P. E., Morales-Rubiano, M. E., \& Ortiz-Riaga, C. (2015). Interacción universidad $\mathrm{y}$ entorno: marco para el emprendimiento. Educación y Educadores, 18(1), 111134.

Secretaría de Educación Pública (2015). Modelo de emprendedores para la educación media superior. Recuperado de http:// www.emprendedores.sems. gob.mx/

Seikkula-Leino, J., Satuvuori, T., Ruskovaara, E., \& Hannula, H. (2015). How Do Finnish Teacher Educators Implement Entrepreneurship Education? Education
+ Training, 57(4), 392404.

Simón, J. D. (2013). Sistematizando experiencias sobre educación en emprendimiento en escuelas de nivel primaria. Revista Mexicana de Investigación Educativa, 18(56), 159-190.

Toca Torres, Claudia. (2010). "Consideraciones para la formación en emprendimiento: Explorando nuevos ámbitos y posibilidades". En: Estudios Gerenciales, 26 (117), 41-60. Fecha de consulta: 04/11/2014. Disponible en: <http://www.scielo.org. $\mathrm{co} /$ scielo.php?script $=$ sci arttext\&pid = S 0123 . 59232010000400003\&lng $=$ en\&tlng $=\mathrm{es}>$

Véliz Capuñay, C. (2017). Análisis multivariante. Métodos estadísticos multivariantes para la investigación. México: Cengage Learning.

Zapata Ros, M. (2015). Teorías y modelos sobre el aprendizaje en entornos conectados y ubicuos. Bases para un nuevo modelo teórico a partir de una visión crítica del "conectivismo". Teoría de la Educación. Educación y Cultura en la Sociedad de la Información, 16(1), 69-102. 